University of Texas Rio Grande Valley

ScholarWorks @ UTRGV

Computer Science Faculty Publications and Presentations

$12-2017$

\title{
The Public's Perception of Humanlike Robots: Online Social \\ Commentary Reflects an Appearance-Based Uncanny Valley, a General Fear of a "Technology Takeover", and the Unabashed Sexualization of Female-Gendered Robots
}

\author{
Megan K. Strait \\ The University of Texas Rio Grande Valley \\ Cynthia Aguillon \\ The University of Texas Rio Grande Valley \\ Virginia Contreras \\ The University of Texas Rio Grande Valley \\ Noemi Garcia \\ The University of Texas Rio Grande Valley
}

Follow this and additional works at: https://scholarworks.utrgv.edu/cs_fac

Part of the Computer Sciences Commons

\section{Recommended Citation \\ M. K. Strait, C. Aguillon, V. Contreras and N. Garcia, "The public's perception of humanlike robots: Online social commentary reflects an appearance-based uncanny valley, a general fear of a "Technology Takeover", and the unabashed sexualization of female-gendered robots," 2017 26th IEEE International Symposium on Robot and Human Interactive Communication (RO-MAN), Lisbon, 2017, pp. 1418-1423, doi: 10.1109/ROMAN.2017.8172490.}

This Conference Proceeding is brought to you for free and open access by the College of Engineering and Computer Science at ScholarWorks @ UTRGV. It has been accepted for inclusion in Computer Science Faculty Publications and Presentations by an authorized administrator of ScholarWorks @ UTRGV. For more information, please contact justin.white@utrgv.edu,william.flores01@utrgv.edu. 


\title{
The Public's Perception of Humanlike Robots: Online Social Commentary Reflects an Appearance-Based Uncanny Valley, a General Fear of a "Technology Takeover", and the Unabashed Sexualization of Female-Gendered Robots
}

\author{
Megan K. Strait ${ }^{1}$, Cynthia Aguillon, Virginia Contreras, and Noemi Garcia
}

\begin{abstract}
Towards understanding the public's perception of humanlike robots, we examined commentary on 24 YouTube videos depicting social robots ranging in human similarity - from Honda's Asimo to Hiroshi Ishiguro's Geminoids. In particular, we investigated how people have responded to the emergence of highly humanlike robots (e.g., Bina48) in contrast to those with more prototypically-"robotic" appearances (e.g., Asimo), coding the frequency at which the uncanny valley versus fears of replacement and/or a "technology takeover" arise in online discourse based on the robot's appearance.

Here we found that, consistent with Masahiro Mori's theory of the uncanny valley, people's commentary reflected an aversion to highly humanlike robots. Correspondingly, the frequency of uncanny valley-related commentary was significantly higher in response to highly humanlike robots relative to those of more prototypical appearances. Independent of the robots' human similarity, we further observed a moderate correlation to exist between people's explicit fears of a "technology takeover" and their emotional responding towards robots. Finally, through the course of our investigation, we encountered a third and rather disturbing trend - namely, the unabashed sexualization of female-gendered robots. In exploring the frequency at which this sexualization manifests in the online commentary, we found it to exceed that of both the uncanny valley and fears of robot sentience/replacement combined. In sum, these findings help to shed light on the relevance of the uncanny valley "in the wild" and further, they help situate it with respect to other design challenges for HRI.
\end{abstract}

\section{INTRODUCTION}

Withing fields concerning the design of artificial agents (e.g., robotics, graphics, animation), there is a relatively pervasive assumption that humanlike agents evoke better responding than do more prototypically-"robotic" (i.e., mechanomorphic) ones. For example, in robotics, this assumption is evident from the sheer number of efforts devoted to engineering increasingly humanlike robots (e.g., [1], [2], [3], [4], [5]) to the instantiation of a new field of study - android science - devoted to this topic [6]. Researchers argue that, by capitalizing on traits that are familiar/intuitive to people, such robots offer more natural and effective interactions than their less humanlike counterparts (e.g., [7]).

This perspective is, in fact, rather well-established by a large (and still growing) empirical base. Equipping a robot with humanlike features facilitates the formation of rapport

\footnotetext{
${ }^{1}$ Department of Computer Science, College of Engineering and Computer Science, The University of Texas Rio Grande Valley; Edinburg, TX USA
}

with, empathy towards, and acceptance of the agent (e.g., [8], [9], [10], [11]). People also attribute humanlike robots better personalities (e.g., [12], [13], [14]) and more positive traits, such as being trustworthy and intelligent (e.g., [15], [16], [17]). Moreover, people report greater comfort in their presence (e.g., [18]). In contrast, mechanomorphic robots are received in a more negative fashion. For example, they are perceived as less intelligent and trustworthy than their more humanlike counterparts (e.g., [19]).

Such observations are broadly consistent with models of psychological interpersonal distancing, such as similarityattraction theory, which predicts that people will respond more positively towards agents the more they resemble themselves (e.g., [20], [21]). With the aim of continued improvement to their acceptance by human interactants, this broad empirical and theoretical support has fed into the development of increasingly anthropomorphic robots.

The emergence of highly anthropomorphized robots, however, has brought to light a counter phenomenon - the uncanny valley [22]. This phenomenon refers to the effect wherein highly humanlike agents provoke discomfort and even aversion in observers (for a review, see [23]). For example, highly humanlike robots are rated more negatively [24], attributed less trustworthiness [17], and avoided more frequently [25], [26] than their less humanlike counterparts.

However, the valley continues to be a relatively contentious topic due, in part, to a paucity of literature as to why the uncanny valley exists (e.g., [27], [28], [29]). In particular, many have asked whether the valley effect can be explained by alternative mechanisms such as a fear of "replacement" or a "technology takeover" (e.g., [30], [31]).

\section{A. Alternative Accounts}

The fear of replacement account, broadly defined, refers to the threat to human identity/distinctiveness from machines that the emergence of increasingly humanlike and capable robots poses. Such fears are more than evident in public discourse regarding the role of robots in society. For example, a Google search with the terms "robot" and "jobs" yields thousands of results with reputable news sources reporting on an "inevitable" loss of jobs due to robots, such as:

- The Telegraph: "Robots will take over most jobs within 30 years" 
- The Guardian: "Robots will eliminate $6 \%$ of all US jobs by $2021 "$

- NBC: "Are humans becoming obsolete in the workforce? All signs point to yes."

- Wired: "The future has lots of robots, few jobs for humans"

- Los Angeles Times: "Robots could take over 38\% of US jobs within about 15 years"

While these headlines are well-overstated, there is empirical evidence that these fears exist and moreover, are exacerbated by the appearance of the robots. In particular, highly humanlike robots have been found to be perceived as a realistic threat to human jobs, safety, and resources, as well as a threat to human identity and uniqueness [30], [32], [33].

The "Technology Takeover" account, on the other hand, refers to the fear of robots becoming sentient and rebelling against humanity. Similarly, this is a common theme in media (particularly in science fiction writing and Hollywood films, though it reaches the academic realms as well: [31]). For example, there's James Cameron's Terminator franchise (19842015), encompassing comics, films, novels, and additional media concerning battles between humans and Skynet, a self-aware synthetic intelligence network. In a similar vein, there's The Matrix (1999-2003), I, Robot (2004), 9 (2009), The 100 television series (2014-), Ex Machina (2015), and so on. This fear also served as a basis for the generation of the purported "laws of robotics" promulgated by the scientist and science fiction author Isaac Asimov [34]. While there is no evidence (yet) that this narrative has translated into actual fears of robots, it is nevertheless an open question for and limitation of the uncanny valley theory [27].

\section{B. The Uncanny Valley in Popular Perception}

Beyond the questions regarding alternative accounts for the theory, there also remains a gap in the literature between in-laboratory studies of the uncanny valley and how it extends to more realistic scenarios with a broader population. This stems in large part due to the methodological challenges involved in bridging that literature gap. In particular, deployment of robots "in the wild" is a substantial technical challenge and furthermore, even obtaining access to more than one robotic platform is a challenge of accessibility (due to cost, limited production, etc.).

There have been a handful of case studies evaluating the perception of androids "in the wild". In particular, several have deployed an android (the Geminoid HI) in public settings, for example with the robot on display as an exhibit [35], portrayed as an innocuous bystander in a cafe [36], and with the robot as a service assistant in a clothing store [37]. All three report relatively successful deployments, with interviewees reporting more positive than negative descriptions of the robot. But, the set of case studies also reported a fairly high frequency of discomfort ( $37.5 \%$ of interviewees reported an uncanny feeling in the interaction), suggesting that even beyond the laboratory (in more comfortable settings), people are uncomfortable to some degree with highly humanlike robots.
It is also worth noting that with case studies, there is limited ability to make general inferences about the effects of anthropomorphism on people's responding. Specifically, with the technical challenges to deployment, it is not practical (or necessarily feasible) to deploy multiple robots for the purposes of comparison. As a result, while case studies provide great insight into how people respond to actual human-robot interactions, they are unable to make general comparisons relating to manipulations of the robots' designs.

\section{Present Work}

We thus conducted an observational study of the effects of a robot's appearance (mechanomorphic or highly anthropomorphized) on people's perceptions. Because of the diversity of robot designs and their limited accessibility, we elected to analyze people's reactions to online videos - an experimental approach that has been adopted previously for larger-scale design investigations (e.g., [38]).

While online and observational methods have their own limitations, this approach offers several advantages. First and foremost, it allows for greater generalizability, as the comparison across many robots (rather than just one, two, or few) reduces influences attributable to any one robot. Secondly, it provides access to a more representative sample by eliminating barriers to investigation and participation (e.g., accessibility of the platforms, availability of testing, geographic location of the experiment, etc.). Finally, the analysis of free-form responses on social media provide a look at people's perceptions, unmoderated by implicit factors in laboratory or direct-observation settings (e.g., an experimenter, conscious observation, etc.). As both the development and deployment of robots in more public venues remain a methodolgical challenge, studies concerning the general design of social robots warrant approaches alternative to the standard in-laboratory experimental study.

In total, we analyzed 1200 comments across 24 distinct videos depicting a robot of either a mechanomorphic or highly humanlike appearance. Comments were coded for the emotional valence of the content and whether they contained references to the uncanny valley and/or fears of replacement or a technology takeover. Based on the evidence outlined here, our hypotheses were as follows:

- H1 (valley hypothesis): people will react more negatively to the highly humanlike robots, as evidenced by the overall valence of their comment (H1a), the frequency at which their commentary refers to the uncanny valley or related concepts ( $\mathrm{H} 1 \mathrm{~b})$, and the correlation between the two (H1c).

- E1 (replacement explanation): fear of replacement (in their jobs, identity, etc.) drives people's negative responding, as evidenced by the frequency at which their commentary refers to it (E1a) and its correlation with the valence of their commentary (E1b).

- E2 (takeover explanation): fear of a "technology takeover" drives people's responding, as evidenced by the reference frequency (E2a) and its correlation with the valence of their commentary (E2b). 
TABLE I

SOURCE INFORMATION OF VIDEOS ANALYZED, BLOCKED BY APPEARANCE CATEGORY (TOP: MECHANOMORPHIC, BOTTOM: HIGHLY HUMANLIKE). $N_{V i e w s}$ INDiCATES THE NUMber (ROUNDED to THE NEAREST THOUSAND) OF VIEWS AND $N_{\text {Likes }}, N_{\text {Dislikes }}$, AND $N_{\text {Comments }}$ INDICATES THE TOTAL NUMBER OF LIKES, DISLIKES, AND COMMENTS WHEN THE VIDEO WAS ACCESSED.

\begin{tabular}{|c|c|c|c|c|c|c|c|}
\hline Robot & Source & Release Year & Video Duration & $N_{\text {Views }}$ & $N_{\text {Likes }}$ & $N_{\text {Dislikes }}$ & $N_{\text {Comments }}$ \\
\hline Asimo & youtu.be/QdQL11uWWcI & 2014 & $3: 58$ & $5,286 K$ & 18,901 & 943 & 2,080 \\
\hline Baxter & youtu.be/gXokWusckRI & 2012 & $3: 23$ & $243 K$ & 425 & 28 & 100 \\
\hline Dreamer & youtu.be/bs j8ixExf7k & 2011 & $3: 03$ & $72 K$ & 162 & 5 & 50 \\
\hline HRP-4 & youtu.be/z-tTmXfUzlQ & 2013 & $4: 35$ & $1,029 K$ & 1,443 & 941 & 418 \\
\hline iCub & youtu.be/ZcTwO2dpX8A & 2011 & $2: 40$ & $139 K$ & 630 & 12 & 90 \\
\hline Kojiro & youtu.be/w3TGMjJLO18 & 2010 & $3: 01$ & $932 K$ & 1,379 & 115 & 1,381 \\
\hline Nao & youtu.be/nNb j2G3GmAo & 2011 & $3: 31$ & $1,496 K$ & 7,973 & 158 & 1,269 \\
\hline Nexi & youtu.be/aQS2zxmrrrA & 2008 & $2: 17$ & $266 K$ & 577 & 20 & 273 \\
\hline Partner & youtu.be/EzjkBwZtxp 4 & 2008 & $1: 39$ & $7,425 K$ & 21,401 & 2,273 & 13,326 \\
\hline Pepper & youtu.be/lqlyxgl-gE0 & 2014 & $2: 13$ & $571 K$ & 1,407 & 75 & 392 \\
\hline PR2 & youtu.be/c3Cq0sy 4 TBs & 2010 & $2: 46$ & $418 K$ & 766 & 16 & 177 \\
\hline Twendy One & youtu.be/CETUmThm8Rg & 2009 & $1: 45$ & $401 K$ & 765 & 37 & 623 \\
\hline Bina48 & youtu.be/G9uLnquaC84 & 2015 & $1: 58$ & $191 K$ & 220 & 58 & 115 \\
\hline Diego & youtu.be/knRyDcnUc $4 \mathrm{U}$ & 2013 & $1: 40$ & $543 K$ & 1,277 & 86 & 337 \\
\hline Geminoid DK & youtu.be/ezlLNVmaPbM & 2011 & $0: 54$ & $2,411 K$ & 2,375 & 75 & 763 \\
\hline Geminoid F & youtu.be/cy 7 xGwYdRk0 & 2012 & $2: 44$ & $1,272 K$ & 2,333 & 140 & 1,343 \\
\hline Geminoid HI & youtu.be/uD1CdjlrTBM & 2012 & $1: 57$ & $149 K$ & 314 & 12 & 78 \\
\hline Han & youtu.be/HJvuzZ-kolo & 2015 & $2: 07$ & $309 K$ & 729 & 45 & 93 \\
\hline $\mathrm{HRP}-4 \mathrm{c}$ & youtu.be/xcZJqiUrbnI & 2010 & $3: 23$ & $2,306 K$ & 3,210 & 232 & 1,630 \\
\hline Jules & youtu.be/xRR33WDFi_k & 2006 & $2: 47$ & $430 K$ & 894 & 51 & 429 \\
\hline Nadine & youtu.be/cvbJGZf-raY & 2015 & $1: 10$ & $302 K$ & 353 & 55 & 293 \\
\hline PKD & youtu.be/t9MUg6uk5lg & 2011 & $1: 36$ & $169 K$ & 293 & 7 & 79 \\
\hline Showa Hanako & youtu.be/WhzbFaNueKU & 2011 & $4: 20$ & $4,748 K$ & 7,000 & 475 & 2,665 \\
\hline Yang Yang & youtu.be/K53t27U1FC0 & 2015 & $3: 56$ & $306 K$ & 342 & 75 & 153 \\
\hline
\end{tabular}

\section{INVESTIGATION}

\section{A. Method}

We conducted an observational, quasi-experimental study of the effects of human similarity of a robot's appearance (two levels; mechanomorphic, versus highly anthropomorphized) on people's perceptions. Towards approximating the public's view of contemporary robots, we analyzed reactions to online videos hosted via the popular platform, YouTube.

\section{B. Materials}

Preliminary Search: Towards obtaining a final set of high quality, highly viewed videos, we first conducted a preliminary search for videos of 40 distinct robots. The specific set of robots searched were decided a priori (based on those tested and identified as such in [25], [26]) with the aim of obtaining a final set of at least 10 robots of each low (mechanomorphic) and high (highly anthropomorphized) human similarity. All videos were obtained on December 2, 2016 via a youtube. com search, with the keywords: [robot name] + "robot". For example, videos of Honda's Asimo robot were searched via the query: "Asimo robot". The results for each query were then filterd by view count (in descending order), and the top result was recorded. ${ }^{1}$ The

\footnotetext{
${ }^{1}$ If a result contained any content that was explicitely negative (e.g., the title, description, verbal content), the video was excluded and the search continued onto considering the next result. For example, the top result for "Nadine robot" (https://youtu.be/ax2w7gz8nd8) was excluded due to its title, "Nadine The Robot Is Amazing And Creepy".
}

information that was recorded during this preliminary search included: a link to the specific video, the year of the video's upload, and the video's view and comment counts.

Video Retention: To ensure the videos were relatively comparable, the 40 initial videos were filtered based on number of comments (any videos with less than 50 comments were excluded), which reduced the set of highly humanlike robots to 12 . To obtain an equal sample size of mechanomorphic robots, we then selected the top 12 most-commented-on videos out of those remaining for the set depicting the more prototypic robots. As a result, we retained a total of 24 videos for the subsequent analyses (see Table I).

\section{Data Acquisition \& Analysis}

For each of the final 24 videos, additional data - specifically, comments - were retrieved from the original source on February 3, 2017. Due to high variation in the number of comments across videos, only the top 50 independent comments were extracted per video. This number was decided a priori to any analysis based on the lowest comment count (78) and the expectation of data loss due to exclusion criteria.

Exclusion Criteria: To further standardize the dataset, comments were excluded if they were not written in english or if they were duplicate/non-independent commentary (e.g., comments embedded within threads or posted as replies to other commentary). In addition, multiple comments by the same user were condensed and treated as one comment if the comments were made sequentially. Otherwise, any 
comments subsequent to a user's first posting were ignored.

Preprocessing: Next, each of the 1200 comments were processed to determine retention for subsequent analysis. Comments that were unrelated to the video content (e.g., on the Baxter video, one person commented: "I am doing STEM!"; on the Dreamer video, another person commented: "vsauce army approaching" in reference to a viral re-direct) or indecipherable (e.g., one person, in response to the HRP-4 video wrote: "Mr.Steal yo gurl") were marked for exclusion and discarded from the data set prior to analysis.

Measures: Each of the remaining comments were then coded on several dimensions by majority agreement amongst three research assistants blind to the hypotheses and conditions. Specifically, comments were coded for the:

- valence of the response (positive, negative, or neutral; Fleiss' $\kappa=.72)$,

- presence ( 0 or 1$)$ of valley-related references $(\kappa=.71)$, such as the explicit mentions of creepiness, uncanniness, or being unsettled,

- presence ( 0 or 1$)$ of replacement-related references $(\kappa=$ .83), such as the explicit mentions of loss of jobs, and

- presence ( 0 or 1$)$ of takeover-related references $(\kappa=$ .83), such as the explicit mentions of apocalyptic themes (e.g., end of humanity, Terminator, etc).

The presence coding was used to compute an overall frequency per video of each of the three themes (the uncanny valley, replacement fear, and fear of a technology takeover) for each of the 24 videos. Lastly, a final dimension frequency of objectification $(\kappa=.88)$, such as explicit references to the performance of sexual acts on or by the robot - was added as an exploratory theme based on its frequent observation early in the coding process.

\section{RESULTS}

We first analyzed the videos for any differences in general qualities (age of video, video duration, number of views/comments, and number of likes/dislikes) between the two appearance categories (mechanomorphic versus highly humanlike). We then tested each of our hypotheses relating to the uncanny valley, followed finally by exploration of the sexualization of robots across appearance categories.

To test for differences, we conducted independent sample $t$ tests (two-tailed, $\alpha=.05$ ) on each of the measures (including video metrics) with human similarity (mechanomorphic versus highly humanlike) as the independent variable.

\section{A. Preliminary Checks}

Between videos depicting mechanomorphic versus highly humanlike robots, there were no significant differences in the age of the videos $(p=.21)$, their duration $(p=.20)$, or the number of: views $(p=.59)$, comments $(p=.39)$, likes $(p=.34)$, and dislikes $(p=.32)$. Thus, these results suggest that there were not any confounding variables, at least as reflected by the objective video statistics.

\section{B. Hypothesis Testing}

Valley Hypothesis (H1): Consistent with our expectations, comments in response to highly humanlike robots were significantly less positive $(M=-.1450, S D=.4809)$ than those in response to robots with more prototypic appearances $(M=.3642, S D=.3405): t=-6.81, p<.01$, Cohen's $d=1.22$. Furthermore, valley-related references appeared more frequently in response to highly humanlike robots ( $M=.2204, S D=.1311$ ) than they did in response to the mechanomorphic robots $(M=.0513, S D=.0657)$ : $t=33.03, p<.01, d=1.63$. See Figure 1 (A-B). Finally, the overall relationship between people's emotional responding (valence of comments) and frequency of uncanny valley-related references is highly significant (Pearson's $r=$ $-.6487, p<.01)$. Taken together, these findings support the valley hypothesis and moreover, suggest that discomfort stemming from the valley effect well predicts people's freeform responding towards humanlike robots.

Alternative Accounts (E1, E2): Two alternative accounts propose that people's fear of replacement (E1) and fear of a technology takeover (E2) may better predict people's responding towards humanlike robots. Testing these proposals, we found there were no differences in the frequency at which people referenced these fears between mechanomorphic robots versus those that are highly humanlike $\left(p_{\text {replacement }}=.7015, p_{\text {takeover }}=.4260\right)$. See Figure 1 (C-D). Furthermore, there was no correlation between comment valence and the frequency at which people referenced a fear of replacement $(p=.1431)$. There was, however, a significant correlation between comment valence and the frequency at which people referenced a technology takeover (Pearson's $r=-.4581, p=.0243$ ). In sum, these findings suggest that there may be a moderate relationship between people's emotional responding to robots and their fear of a robot apocalypse. Nevertheless, such fears do not seem to drive the differential responding towards robots of varying human similarity.

\section{Exploratory Analyses}

We lastly considered a trend that emerged in reviewing the video commentary: the objectification of robots, particularly those with explicit cues gendering them as female. For this, we conducted three comparisons: between appearance categories and then, within each category but between genders.

All comparisons were significant. Specifically, people objectified highly humanlike robots more frequently $(M=$ $.2409, S D=.2587$ ) relative to the mechanomorphic robots $(M=.0604, S D=.0420): t=9.34, p=.03, d=.97$. However, people were highly selective in their objectification with female-gendered robots receiving significantly more dehumanizing comments than did the neutral- and male-gendered robots within both the set of highly humanlike robots $\left(M_{\text {female }}=.4405, S D=.2241 ; M_{\text {male }}=\right.$ $.0414, S D=.0379 ; t=19.46, p<.01, d=2.48)$, as well as marginally within the set of mechanomorphic robots $\left(M_{\text {female }}=.0944, S D=.0448 ; M_{\text {male }}=.0434, S D=\right.$ $.0303 ; t=48.37, p=.10, d=1.33)$. See Figure $1(\mathrm{E})$. 

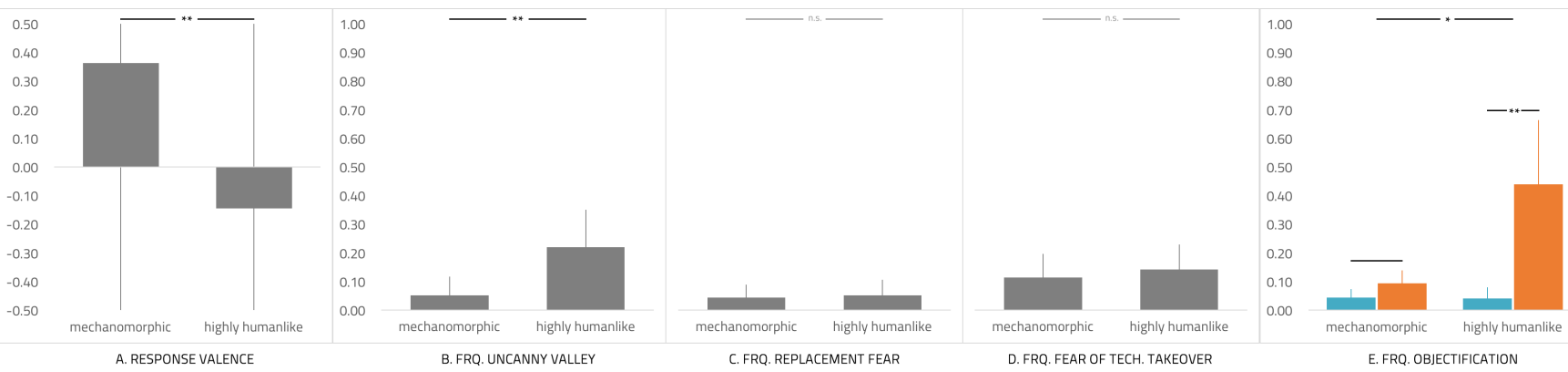

C. FRQ. REPLACEMENT FEAR

D. FRQ. FEAR OF TECH. TAKEOVER

E. FRQ. OBJECTIFICATION

Fig. 1. Hypothesis Testing: effect of robot appearance on (A) valence of user commentary (left), as well as the frequency of references to the (B) uncanny valley (middle-left), (C) a replacement fear (middle-center), and (D) fear of a technology takeover (middle-right). (E) The frequency of objectifying commentary (right) by robot appearance and gender (blue: male- or $a$-gendered robots; orange: robots explicitly gendered as female). Bars indicate pairwise comparisons tested and asterisks denote the significance level $(*: p<.05 ; * *: p<.01 ; n . s .:$ non-significant).

\section{Discussion}

\section{A. Summary of Findings \& General Implications}

Consistent with recent uncanny valley literature (e.g., [23], [24], [25]), participants exhibited clear discomfort in response to the highly humanlike robots, as evidenced by the negative-on-average commentary and frequency of uncanny valley-related commentary. Moreover, the valence of people's comments were best predicted by the frequency of valleyrelated references (as opposed to the relationship with people's fears of replacement/a technology takeover). As such, these findings further underscore the need for attention to the uncanny valley in the design of humanlike robots.

In contrast, despite an abundance of catchy headlines reporting an "inevitable" replacement of humans by robots and captivating films centered on the theme of a "robot apocolypse", the frequencies at which people referenced alternative sources of discomfort were unaffected by the robots' appearances. Furthermore, relative to the frequency of valley-related concepts, such fears were not particularly prevalent in the general commentary. We did, however, find an overall correlation between the frequency at which people mentioned fears of a "technology takeover" and people's emotional responding to the videos. This suggests, rather surprisingly, that the idea of a robot apocalypse - something that exists only within science fiction - negatively affects people's perceptions of actual robots and robotic technologies.

Finally, in the process of conducting the present research, a disturbing observation was made regarding the frequency at which robots with explicit cues gendering them as female are sexualized. Such comments ranged from rather direct (e.g., "Can you fuck it?") to more subtle, albeit not by much (e.g., "The only problem with these is they'll need to replace them monthly due to semen corrosion."). Moreover, towards half of the top comments on videos depicting a femalegendered robot are objectifying in nature, which exceeds the frequencies of all other themes combined.

As gender-based stereotyping has been observed to extend to human-robot interaction (e.g., [38]), the presence of objectifying commentary is not in itself so surprising. However, the propensity of commentators to respond to superficial cues of gender in this manner and so unabashedly so, indicates a profound gap between the public's responding versus what was likely intended by researchers.

Such reactions raise several questions warranting further investigation. Amongst others, understanding the psychology behind the objectification of robots appears valuable as a design-related pursuit, especially considering the fact that superficial design factors (cueing perceptions of female-ness) prompted people's engagement in abusive commentary. Too, as these robots are intended for social settings, it is worth asking how we might be proactive in engineering the robots themselves to be responsive to inappropriate interactions.

\section{B. Limitations \& Avenues for Future Research}

The present work contributes a novel look at the uncanny valley and situates it relative to other concerning topics. Furthermore, it uncovers a significant issue related to the gendering of robots (as female) in their design/appearance. Together, the findings hold implications for the design of social robots. Nevertheless, the study has several limitations which underscore important avenues for further research. In particular, the lack of demographic information (a limitation inherent in online-based methodologies) of those participating in the video commentary constrains the conclusions that can be drawn here. Specifically, it remains unknown whether the present population is well-representative of the general public. As YouTube is a popular media platform, we conclude that they are representative of consumers of online information. However, it is likely that this population skews towards a homogenous group that is largely white and male, and therefore more likely to be objectifying of femalegendered agents ${ }^{2}$. More generally, socio-demographic factors (e.g., age, gender, etc.) can impact perceptions of humanrobot interaction (e.g., [39], [40], [41], [42]). As such, subsequent investigation of a more controlled nature is necessary to confirm the trends identified in the present work.

\section{CONCLUSIONS}

Here we aimed to sample the public's perception of social robots, particularly those that are highly humanlike. Specifically, we measured the frequency at which people

${ }^{2}$ See for example: https://goo.gl/T7g4Px. 
communicated discomfort in response to a robot and analyzed whether their discomfort stemmed from one of three explanatory mechanisms (the uncanny valley, replacement fear, and fear of a "technology takeover"). Our results show that highly humanlike robots elicit significantly more discomfort and commentary relating to the uncanny valley. The degree of the robots' anthropomorphism, however, had no effect on the frequency at which fears of replacement and of a technology takeover were referenced. In addition, we made a disturbing discovery: namely, the frequency at which the people sexualized the female-gendered robots eclipsed that of all other concepts examined. These findings underscore both a need for careful attention to not only how people perceive humanlike robots, but also how people react. Specifically, our results suggest that the uncanny valley is a relevant concern in how the public receives emerging systems. In addition, they suggest that gendering robots as female may have significant consequences for the efficacy of interactions between people and robots.

\section{REFERENCES}

[1] K. Dautenhahn, C. L. Nehaniv, M. L. Walters, B. Robins, H. KoseBagci, N. A. Mirza, and M. Blow, "Kaspar-a minimally expressive humanoid robot for human-robot interaction research," Applied Bionics and Biomechanics, 2009.

[2] G. Metta, G. Sandini, D. Vernon, L. Natale, and F. Nori, "The iCub humanoid robot: an open platform for research in embodied cognition," in Workshop on Performance Metrics for Intelligent Systems, 2008.

[3] K. Ogawa, S. Nishio, K. Koda, K. Taura, T. Minato, C. T. Ishii, and H. Ishiguro, "Telenoid: tele-presence android for communication," in ACM Conference on Emerging Technologies, 2011.

[4] J. Oh, D. Hanson, W. Kim, I. Y. Han, J. Kim, and I. Park, "Design of android type humanoid robot Albert HUBO," in IROS, 2006, pp. $1428-1433$.

[5] D. Sakamoto, T. Kanda, T. Ono, H. Ishiguro, and N. Hagita, "Android as a telecommunication medium with a human-like presence," in ACM/IEEE HRI, 2007.

[6] H. Ishiguro, "Android science: conscious and subconscious recognition," Connection Science, 2006.

[7] B. R. Duffy, "Anthropomorphism and the social robot," Robots \& Autonomous Systems, 2003.

[8] S. Andrist, X. Z. Tan, M. Gleicher, and B. Mutlu, "Conversational gaze aversion for humanlike robots," in ACM/IEEE HRI, 2014.

[9] A. Kupferberg, S. Glasauer, M. Huber, M. Rickert, A. Knoll, and T. Brandt, "Biological movement increases acceptance of humanoid robots as human partners in motor interaction," AI \& society, 2011.

[10] L. D. Riek, T.-C. Rabinowitch, B. Chakrabarti, and P. Robinson, "How anthropomorphism affects empathy toward robots," in ACM/IEEE HRI, 2009.

[11] M. Strait, C. Canning, and M. Scheutz, "Investigating the effects of robot communication strategies in advice-giving situations based on robot appearance, interaction modality and distance," in ACM/IEEE HRI, 2014.

[12] S. Andrist, B. Mutlu, and A. Tapus, "Look like me: matching robot personality via gaze to increase motivation," in ACM CHI, 2015.

[13] E. Broadbent, V. Kumar, X. Li, J. Sollers, R. Q. Stafford, B. A. MacDonald, and D. M. Wegner, "Robots with display screens: a robot with a more humanlike face display is perceived to have more mind and a better personality," PloS one, 2013.

[14] T. Koda and P. Maes, "Agents with faces: The effect of personification," in IEEE RO-MAN, 1996.

[15] K. Gray and D. M. Wegner, "Feeling robots and human zombies: Mind perception and the uncanny valley," Cognition, 2012.

[16] F. Hegel, S. Krach, T. Kircher, B. Wrede, and G. Sagerer, "Understanding social robots: A user study on anthropomorphism," in IEEE RO-MAN, 2008.

[17] M. B. Mathur and D. B. Reichling, "Navigating a social world with robot partners: A quantitative cartography of the uncanny valley," Cognition, 2016.
[18] A. Sauppé and B. Mutlu, "The social impact of a robot co-worker in industrial settings," in ACM CHI, 2015.

[19] C. Bartneck and J. Hue, "Exploring the abuse of robots," Interaction Studies, 2008.

[20] C. R. Berger and R. J. Calabrese, "Some explorations in initial interaction and beyond: Toward a developmental theory of interpersonal communication," Human Communication Research, 1975.

[21] N. Eisenberg and P. A. Miller, "The relation of empathy to prosocial and related behaviors," Psychological Bulletin, 1987.

[22] M. Mori, "Bukimi no tani [the uncanny valley]," Energy, 1970, translated by K. F. MacDorman and N. Kageki (2012), IEEE Robotics and Automation Magazine 19, 98-100.

[23] J. Kätsyri, K. Förger, M. Mäkäräinen, and T. Takala, "A review of empirical evidence on different uncanny valley hypotheses: support for perceptual mismatch as one road to the valley of eeriness," Frontiers in Psychology, 2015.

[24] K. F. MacDorman, "Subjective ratings of robot video clips for human likeness, familiarity, and eeriness: An exploration of the uncanny valley," in COGSCI, 2006.

[25] M. Strait, L. Vujovic, V. Floerke, M. Scheutz, and H. L. Urry, "Too much humanness for human-robot interaction: exposure to highly humanlike robots elicits aversive responding in observers," in $A C M$ CHI, 2015.

[26] M. K. Strait, V. A. Floerke, W. Ju, K. Maddox, J. Remedios, M. F. Jung, and H. L. Urry, "Understanding the uncanny: Both atypical features and category ambiguity provoke aversion towards humanlike robots," Frontiers in Psychology, 2017.

[27] D. Hanson, A. Olney, S. Prilliman, E. Mathews, M. Zielke, D. Hammons, R. Fernandez, and H. Stephanou, "Upending the uncanny valley," in $A A A I, 2005$.

[28] S. Wang, S. O. Lilienfeld, and P. Rochat, "The uncanny valley: Existence and explanations," Review of General Psychology, 2015.

[29] J. Zlotowski, D. Proudfoot, and C. Bartneck, "More human than human: does the uncanny curve really matter?" 2013.

[30] F. Ferrari, M. P. Paladino, and J. Jetten, "Blurring human-machine distinctions: Anthropomorphic appearance in social robots as a threat to human distinctiveness," International Journal of Social Robotics, 2016.

[31] B. Kirman, C. Linehan, S. Lawson, and D. O'Hara, "Chi and the future robot enslavement of humankind: a retrospective," in ACM CHI, 2013.

[32] K. Yogeeswaran, J. Złotowski, M. Livingstone, C. Bartneck, H. Sumioka, and H. Ishiguro, "The interactive effects of robot anthropomorphism and robot ability on perceived threat and support for robotics research," Journal of Human-Robot Interaction, 2016.

[33] J. Złotowski, K. Yogeeswaran, and C. Bartneck, "Can we control it? autonomous robots threaten human identity, uniqueness, safety, and resources," International Journal of Human-Computer Studies, 2017.

[34] P. Hancock, D. Billings, and K. Schaefer, "Can you trust your robot?" Ergonomics in Design: The Quarterly of Human Factors Applications, 2011.

[35] C. Becker-Asano, K. Ogawa, S. Nishio, and H. Ishiguro, "Exploring the uncanny valley with Geminoid HI-1 in a real-world application," in Conference on Interfaces and Human Computer Interaction, 2010.

[36] A. M. Rosenthal-von der Pütten, N. C. Krämer, C. Becker-Asano, K. Ogawa, S. Nishio, and H. Ishiguro, "The uncanny in the wild: Analysis of unscripted human-android interaction in the field," International Journal of Social Robotics, 2014.

[37] M. Watanabe, K. Ogawa, and H. Ishiguro, "Can androids be salespeople in the real world?" in $A C M$ CHI, 2015.

[38] J. Otterbacher and M. Talias, "S/he's too warm/agentic!: The influence of gender on uncanny reactions to robots." in ACM/IEEE HRI, 2017.

[39] I. H. Kuo, J. M. Rabindran, E. Broadbent, Y. I. Lee, N. Kerse, R. Stafford, and B. A. MacDonald, "Age and gender factors in user acceptance of healthcare robots," in IEEE RO-MAN, 2009.

[40] H. R. Lee and S. Sabanović, "Culturally variable preferences for robot design and use in south korea, turkey, and the united states," in ACM/IEEE HRI, 2014.

[41] M. Strait, P. Briggs, and M. Scheutz, "Gender, more so than age, modulates positive perceptions of language-based human-robot interactions," in International Symposium on New Frontiers in HumanRobot Interaction, 2015.

[42] S. S. Sundar, T. F. Waddell, and E. H. Jung, "The hollywood robot syndrome: media effects on older adults' attitudes toward robots and adoption intentions," in ACM/IEEE HRI, 2016. 